\title{
Efficient Synthesis of Spirolactams by Ring-Closing Olefin Metathesis
}

\author{
Seok Won Kang ${ }^{+*}$ and Sung Hoon Kim ${ }^{*}+$ \\ "Department of Organic Synhesis Research, Jeil Pharmacentical Co., Ltt., Yongin, Gyeonggi 449-861, Korea \\ "Department of Chemistr, Konkuk University, Seoul 143-701, Korea. *E-mail: shkim(tikonktkac.kr. \\ Received November 7, 2005
}

Key Words : Spirolactam, Ring-closing olefin metathesis, RCM

Spirobicyclic cores play an important role in the development of new bioactive substances. ${ }^{1}$ This type of spiro system exists as a key framework of numerous steroids. ${ }^{2}$ Construction of spirocyclics can be achieved by intramolecular alkylation, ${ }^{3}$ cycloaddition reaction, ${ }^{4}$ radical cyclization ${ }^{5}$ and ring-closing metathesis reaction. ${ }^{6}$ Various approaches for the preparation of spirocyclics encountered problems related with functional group incompatibility at one or more stages, and they have been restricted to a single substitution pattern. Only in a few instances, the newly generated ring system was left with useful functional groups for further transformations. Therefore, there has been a continuous need to develop new methods for the preparation of spiro-linkage with additional functionality for further synthetic manipulation under mild condition.

In this paper, we intend to report an efficient and versatile synthetic method for the preparation of spirolactams which involves zinc mediated allylation of cyclic imides followed by ring-closing metathesis using Grubbs catalyst. ${ }^{7}$ We previously reported that gem-diallylated precursors $\mathbf{3 a - b}$ can be prepared efficiently from cyclic imides $\mathbf{1} \mathbf{a}-\mathbf{b} .{ }^{\$}$ Babier-type zinc mediated allylation of cyclic imides $\mathbf{1 a - b}$ with allyl bromide in the presence of catalytic amount of $\mathrm{PbBr}_{2}$ gave hydroxylactams $\mathbf{2 a - b}$. Subsequent allylation of $\mathbf{2 a - b}$ with allyltributyltin in the presence of $\mathrm{BF}_{3} \cdot \mathrm{OEt}_{2}$ afforded precursors 3a-b in good overall yield (Scheme l).<smiles>O=C1CCC(=O)N(Cc2ccccc2)C1=O</smiles>

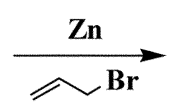

1a,b

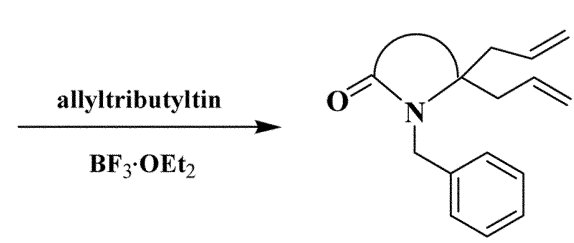

3a,b

Scheme

Next, we utilized this reaction to transform it into the corresponding spirolactams in an excellent yield by intramolecular ring-closing olefin metathesis." The RCM reaction was performed using $3 \mathrm{~mol} \%$ of Grubbs ruthenium catalyst $\left[\left(\mathrm{Cy}_{3} \mathrm{P}\right)_{2} \mathrm{Cl}_{2} \mathrm{Ru}=\mathrm{ClI}-\mathrm{Ph}\right] . \mathrm{After}$ stirring in dichloromethane at $35^{\circ} \mathrm{C}$ under $\mathrm{Ar}$ atmosphere for $4 \mathrm{hr}$, the cyclized products were isolated in high yield (Scheme 2 ).

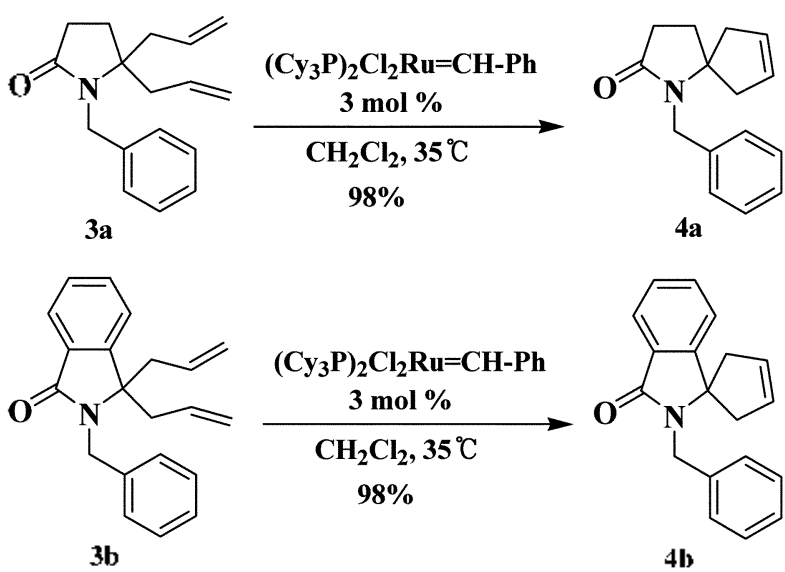

Scheme 2

The results are summarized in Table 1. Structures of the spirolactams were assigned on the basis of ${ }^{1} \mathrm{H} N M R$ and ${ }^{13} \mathrm{C}$.

Table 1. Allylation of cyclic imides and ring-closing metathesis of diallyl lactams

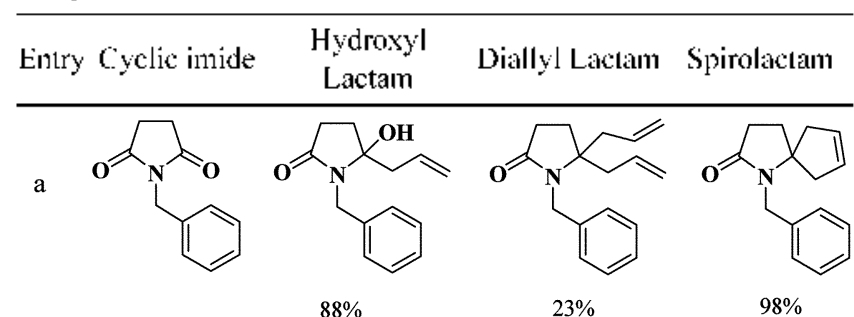

b
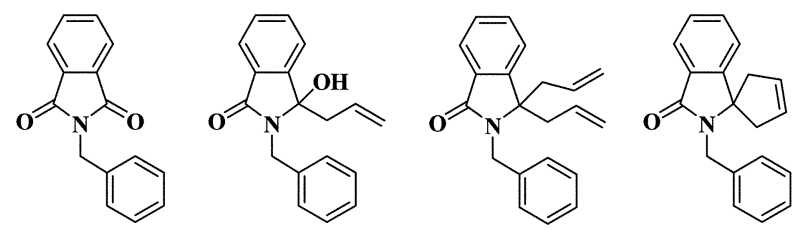

$94.5 \%$

$98 \%$

$98 \%$ 
NMR spectroscopic data.

In conclusion, we have demonstrated that diallyl lactams can be utilized in conjunction with ring-closing metathesis as an efficient method for construction of spirolactams. This RCM method as developed in this study offers very mild conditions for direct formation of spirolactams in an excellent yield. The methodology described herein may lead to interesting applications in synthesis of complex natural and non-natural products due to the presence of double bond and carbonyl group for further functionalization.

\section{Experimental Section}

5-Allyl-1-benzyl-5-hydroxy-2-pyrrolidinone (2a). To a mixture of $N$-benzylsuccinimide ( $5 \mathrm{mmol}$ ), zinc granule (10 mmol) and catalytic amount of $\mathrm{PbBr}_{2}$ in $\mathrm{THF}(10 \mathrm{~mL})$ was added ally bromide $(10 \mathrm{mmol})$ slowly at $0-5^{\circ} \mathrm{C}$, stirred for 5 hr until it became sticky greenish gray slurry. The reaction mixture was quenched with aq. $\mathrm{NH}_{4} \mathrm{Cl}$ solution $(30 \mathrm{~mL})$, extracted with methylene chloride $(2 \times 50 \mathrm{~mL})$. The methylene chloride solution was washed with brine $(10 \mathrm{~mL})$, dried over sodium sulfate, filtered and concentrated to give almost pure 2 a $(88 \%)$ which was crystallized out from ether/hexane to give as a white solid: $\mathrm{mp} 88^{\circ} \mathrm{C}$; ${ }^{1} \mathrm{H}$ NMR $(400 \mathrm{MHz}$, $\left.\mathrm{CDCl}_{3}\right) \delta 7.1-7.3(\mathrm{~m}, 5 \mathrm{H}), 5.4-5.5(\mathrm{~m}, 2 \mathrm{H}), 4.9-5.0(\mathrm{~m}, 2 \mathrm{H})$, $4.6(\mathrm{~d}, 1 \mathrm{H}), 4.3(\mathrm{~d}, 1 \mathrm{H}), 2.4(\mathrm{~m}, 2 \mathrm{H}), 1.9-2.2(\mathrm{~m}, 4 \mathrm{H}) ;{ }^{13} \mathrm{C}$ NMR $\left(100 \mathrm{MHz}, \mathrm{CDCl}_{3}\right) \delta 176.2,138.7,132.4,128.8$, $128.4,127.5,119.7,92.4,44.4,42.8,32.2,29.5$.

3-Allyl-2-benzyl-3-hydroxy-2,3-dihydroisoindol-1-one (2b). mp 144-145 ${ }^{\circ} \mathrm{C}$; ${ }^{1} \mathrm{H}$ NMR (400 MHz, $\mathrm{CDCl}_{3}$ ): $\delta 7.59$ (d, $1 \mathrm{H}), 7.46-7.13(\mathrm{~m}, 8 \mathrm{H}), 5.01(\mathrm{~m}, 1 \mathrm{H}), 4.70(\mathrm{dd}, 1 \mathrm{H}), 4.68$ (dd, 1H), $4.42(\mathrm{q}, 2 \mathrm{H}), 3.51(\mathrm{~s}, 1 \mathrm{H}), 2.72(\mathrm{dd}, 1 \mathrm{H}), 2.55$ (dd, $1 \mathrm{H}) ;{ }^{13} \mathrm{C} \mathrm{NMR}\left(100 \mathrm{MHz}, \mathrm{CDCl}_{3}\right): \delta 167.9,146.7,138.5$, $132.4,131.0,130.8,129.7,128.7,128.6,127.5,123.5$, $122.5,119.7,91.1,425.2,41.3$.

3,3-Diallyl-2-benzyl-2,3-dihydroisoindol-1-one (3b). To a solution of $2 \mathrm{~b}(5 \mathrm{mmol})$ in methylene chloride $(25 \mathrm{~mL})$, under an argon atmosphere at $-78^{\circ} \mathrm{C}$ was added allyltributyltin followed by $\mathrm{BF}_{3} \mathrm{OEt}_{2}$ dropwise. The reaction mixture was quenched with $5 \% \mathrm{NaHCO}_{3}$ solution $(30 \mathrm{~mL})$, then extracted with methylene chloride $(2 \times 50 \mathrm{~mL})$. The organic layer was washed with brine $(10 \mathrm{~mL})$, dried over sodium sulfate, filtered and concentrated to give almost pure diallylated product 3b (98\%): mp 92-93 ' C; 'H NMR (400 $\left.\mathrm{MHz}, \mathrm{CDCl}_{3}\right) \delta 7.4(\mathrm{~m}, 4 \mathrm{H}), 7.2(\mathrm{~m}, 5 \mathrm{H}), 4.9(\mathrm{~m}, 2 \mathrm{H}), 4.6$ (d, $2 \mathrm{H}), 4.5(\mathrm{~m}, 4 \mathrm{H}), 2.5(2,4 \mathrm{H}) ;{ }^{13} \mathrm{C}$ NMR $(100 \mathrm{MHz}$, $\left.\mathrm{CDCl}_{3}\right) \delta 169.5,147.8,138.7,132.5,132.0,131.4,129.4,129.1$,
$128.8,128.5,127.8,122.1,121.1,119.5,69.4,43.4,41.9$.

5,5-Diallyl-1-benzylpyrrolidin-2-one (3a). mp $88{ }^{\circ} \mathrm{C} ;{ }^{1} \mathrm{H}$ NMR (400 MHz, $\left.\mathrm{CDCl}_{3}\right): \delta 7.27-7.13(\mathrm{~m}, 5 \mathrm{H}), 5.4(\mathrm{~m}, 2 \mathrm{H})$, $4.95(\mathrm{dd}, 4 \mathrm{H}), 4.3(\mathrm{~d}, 1 \mathrm{H}), 4.4(\mathrm{~s}, 2 \mathrm{H}), 2.3(\mathrm{~m}, 2 \mathrm{H}), 2.2(\mathrm{~m}$, $2 \mathrm{H}), 2.0(\mathrm{~m}, 2 \mathrm{H}), 1.8(\mathrm{~m}, 2 \mathrm{H}) ;{ }^{13} \mathrm{C} \mathrm{NMR}\left(100 \mathrm{MHz}, \mathrm{CDCl}_{3}\right)$ : $\delta 176.2,139.0,132.7,128.8,128.6,127.6,119.7,72.2,66.4$, $43.9,43.6,30.2,27.8$.

1-Benzyl-1-azaspiro[4.4]non-7-en-2-one (4a). Catalytic amount of $(3 \mathrm{~mol} \%)$ Grubbs ruthenium catalyst $\left[\left(\mathrm{Cy}_{3} \mathrm{P}\right)_{2}-\right.$ $\left.\mathrm{Cl}_{2} \mathrm{Ru}=\mathrm{CH}-\mathrm{Ph}\right]$ was added to the solution of the diallyl lactam $3 \mathrm{a}(0.7 \mathrm{mmol})$ in dichloromethane $(4 \mathrm{~mL})$ at $\mathrm{rt}$. The reaction mixture was stirred for $4 \mathrm{hr}$ at $35^{\circ} \mathrm{C}$ under $\mathrm{N}_{2}$. At the end of the reaction (TLC monitoring), the solvent was removed under reduced pressure and the crude product was purified by flash chromatography using hexane-EtOAc ( 1 : 1 ) as an eluent to give spirolactam $4 \mathrm{a}$ as a white solid (98\%): 'H NMR (400 MHz, $\mathrm{CDCl}_{3}$ ): $\delta 7.21-7.29(\mathrm{~m}, 5 \mathrm{H}), 5.63(\mathrm{~s}$, $2 \mathrm{H}), 4.39(\mathrm{~s}, 2 \mathrm{H}), 2.45-2.51(\mathrm{~m}, 4 \mathrm{H}), 2.28-2.32(\mathrm{~d}, 2 \mathrm{H})$, $2.08-2.12(\mathrm{t}, 2 \mathrm{H}) ;{ }^{13} \mathrm{C} \mathrm{NMR}\left(100 \mathrm{MHz}, \mathrm{CDCl}_{3}\right): \delta 175.7$, $139.0,129.2,128.7,127.7,127.3,70.5,43.9,43.7,35.6,30.4$.

Similarly, the reaction of the diallyl lactam $3 \mathbf{b}$ afforded spirolactam $4 \mathrm{~b}$ in $98 \%$ yield: 'H NMR ( $\left.400 \mathrm{MHz} \mathrm{CDCl}_{3}\right) ; \delta$ $7.85-7.87(\mathrm{~d}, 1 \mathrm{H}), 7.22-7.53(\mathrm{~m}, 8 \mathrm{H}), 5.79(\mathrm{~s}, 2 \mathrm{H}), 4.64(\mathrm{~s}$, $2 \mathrm{H}), 2.51-2.76(\mathrm{dd}, 4 \mathrm{H}) ;{ }^{13} \mathrm{C} \mathrm{NMR}\left(100 \mathrm{MHz}_{2} \mathrm{CDCl}_{3}\right): \delta$ $168.2,152.1,138.9,132.4,131.0,129.5,128.6,128.35$, $128.34,27.5,123.8,121.1,71.3,44.1,43.5$.

Acknowledgements. This work was financially supported by Konkuk university 2004 .

\section{References}

I. (a) Boivin, T. L. B. Tetrahedron 1987, 43, 3309. (b) Kotha, S. Deb, A. S.; Kumar, R. V. Bioorg. Med. Chem. Lett. 2005, 15, 1039.

2. (a) Kende, A. S.; Hernando, J. J. M.; Milbank, J. B. J. Tetrahedron 2002, 58, 6l. (b) Suga, S.; Watanabe, M.; Yoshida, J. I. J. Am. Chem. Soc. 2002, 124, 14824.

3. (a) Martin, S. F. Tetrahedron 1980, 36, 419. (b) Srikrishna, A.; Kumar, P. V.; Viswajanani, R. Tetrahedron Lett. 1996, 37, 1683.

4. Krapcho, A. P. Synthesis 1978, 77.

5. Srinivasan, R.; Rajagopalan, K. Tetrahedron 1997, 53, 11685.

6. Hughes, R. C.; Dvorak, C. A.; Meyers, A. I. J. Org. Chem. 2001, $66(16), 5545$.

7. (a) Grubbs, R. H.; Chang, S. B. Tetrahedron 1998, 54, 4413. (b) Brimble, M. A.; Trzoss, M. Tetrahedron 2004, 60, 5613. (c) Grubbs, R. H. Tetrahedron 2004, 60,7117.

8. Kang. S. W.; Heo. E. Y.; Jun. J. G; Kim. S. H. Bull. Korean Chent. Soc. 2004, 25(12), 1924.

9. Mathias, S.; Siegfried, B. Angew. Chem. Int. Ed. Engl. 1997, 36, 2036. 